\title{
Musical acoustics of orchestral water crotales
}

\author{
Randy Worland ${ }^{\text {a) }}$ \\ Department of Physics, University of Puget Sound, 1500 North Warner, Tacoma, Washington 98416-1031
}

(Received 31 October 2010; revised 13 March 2011; accepted 15 March 2011)

\begin{abstract}
An experimental investigation of orchestral crotale vibrational modes in water is presented, along with a qualitative virtual mass model describing the observed effects. Changes in frequency, overtone ratio, and mode splitting as a function of water depth are reported for a $C_{6}$ crotale using electronic speckle-pattern interferometry. These data are related to perceived changes in pitch and timbre, along with the creation of audible beats at particular water depths. It is also shown that the suspension method used by musicians when dipping crotales into water leads to the creation of additional acoustically significant modes that are not excited when the crotales are mounted in the standard manner. (C) 2012 Acoustical Society of America. [DOI: 10.1121/1.3651228]
\end{abstract}

PACS number(s): 43.75.Kk, 43.40.Dx [NHF]

Pages: 935-944

\section{INTRODUCTION}

Orchestral crotales are small bronze cymbals shaped like disks with an added central boss [see Fig. 1(a)]. Each crotale produces a definite pitch when struck with a hard mallet. The typical set of crotales encompasses two chromatic octaves, sounding from $C_{6}$ to $C_{8}$ (equivalent to the top two octaves of the piano), although one manufacturer ${ }^{1}$ sells a $21 / 2$ octave set extending to $F_{8}$. Contemporary composers writing for percussion often incorporate unconventional playing techniques with the use of traditional instruments. Among these extended techniques is the lowering of crotales into water as they are being struck, resulting in a glissando effect that involves changes in both pitch and timbre, as well as the creation of beats at some water depths. Crotales are traditionally mounted on posts through the center hole, laid out in the pattern of notes on a keyboard instrument [see Fig. 1(b)], but are usually suspended individually by string through the center hole when being lowered into water [see Fig. 1(c)]. This change in support introduces additional vibrational modes as shown in Sec. III.

The origins of the water crotale technique date back to the use of gongs in water by the American avant-garde composer John Cage ${ }^{2}$ in the early 1940s. Since that time, water crotales have been called for in a variety of musical genres including film scores $^{3}$ and percussion ensemble pieces. ${ }^{4}$ Although the water gong effect is subtle and often somewhat hidden among the sounds of a large ensemble, an exposed section of water crotales can be heard at the end of the first movement of American composer David Maslanka's Symphony No. 2 for Wind Ensemble. ${ }^{5}$

Existing literature on the physics of crotales is limited to their operation in air, as traditionally played. $^{6,7}$ Most recently, the physics of orchestral crotales played in the conventional manner has been reported in this journal. ${ }^{8}$ The current work verifies and extends the work of Deutsch et al. by investigating the first ten crotale modes, both in air and water.

\footnotetext{
a) Author to whom correspondence should be addressed. Electronic mail: worland@pugetsound.edu
}

Studies of disk vibrations in the presence of fluids date back to Rayleigh's calculation of the increase in inertia of a vibrating rigid circular plate due to the presence of air. ${ }^{9}$ Lamb's classic paper ${ }^{10}$ of 1920 on the vibrations of a clamped plate in contact with water on one side provides the starting point for most modern studies of plate vibrations in water. Lamb calculated the effect of the added inertia of the water on the vibrational frequencies of the lowest two clamped plate modes, and also showed that the additional damping caused by the water does not play a significant role in the frequency change. McLachlan ${ }^{11}$ extended Lamb's calculations to include vibrations of free-edge disks for modes with any number of nodal diameters.

The engineering literature includes numerous additional works on the natural frequencies of plates in water (i.e., the fluid-structure interaction problem). ${ }^{12-16}$ These include theoretical, numerical, and experimental studies of mode shapes and frequencies, dealing primarily with flat plates, as opposed to the crotale geometry. Much, though not all, of this work emphasizes cases with clamped boundaries as well. In addition, most of these papers address the problem of a disk with water fully covering either one or both sides. We are aware of only one paper, due to Jeong et al., ${ }^{15}$ that treats the variation of frequencies of a partially submerged plate as a function of water depth, analogous to the water crotale effect. The work of Jeong et al. is a theoretical and numerical analysis of plates with clamped boundaries, with one side wet.

None of the previously reported work covers the geometry and boundary conditions of the water crotale, and more importantly, none addresses the results in the context of the musical sound that results when objects such as percussion instruments are submerged in water. This paper provides experimental data and analysis of crotale modes as a function of water depth, with emphasis on the musically relevant aspects of the resulting sound.

\section{CROTALE PHYSICS}

The crotale is essentially a circularly symmetric disk, with a free edge. For such disks the spatial part of the normal mode eigenfunctions may be written as 


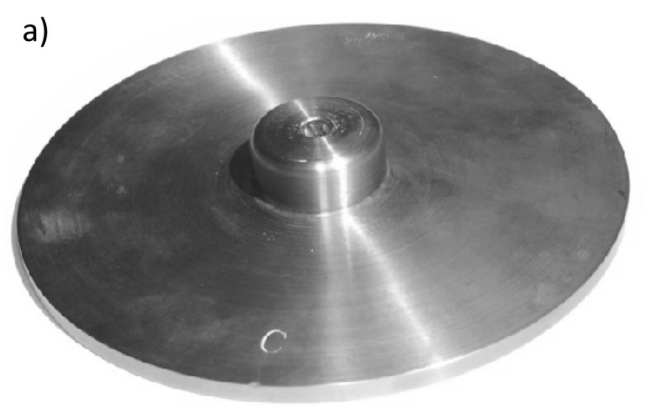

b)

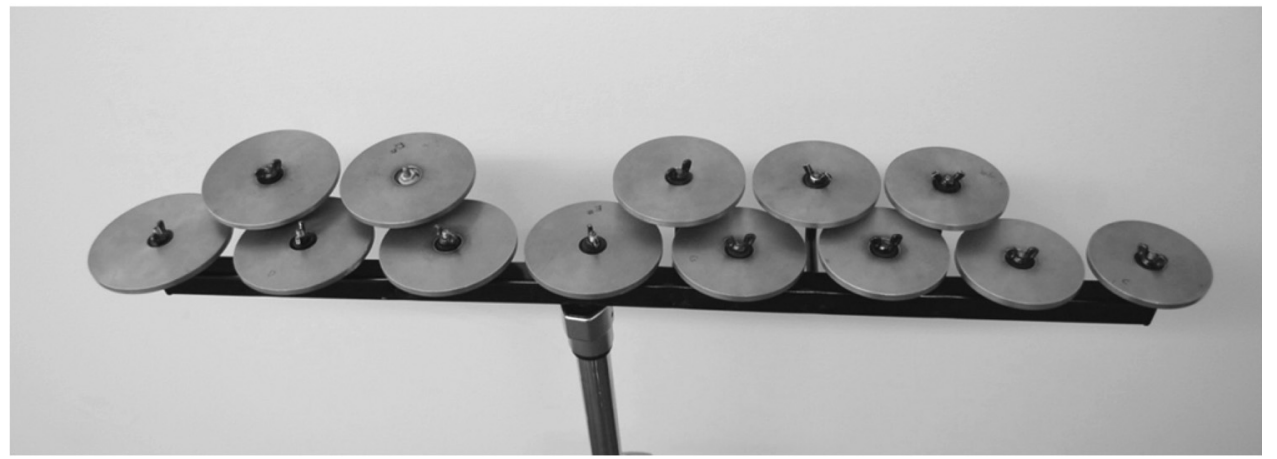

FIG. 1. (a) A Zildjian $C_{6}$ crotale. (b) One octave Zildjian crotale set mounted on a conventional stand, with pitches arranged as on a keyboard instrument. (c) $C_{6}$ crotale suspended in water with string.

c)

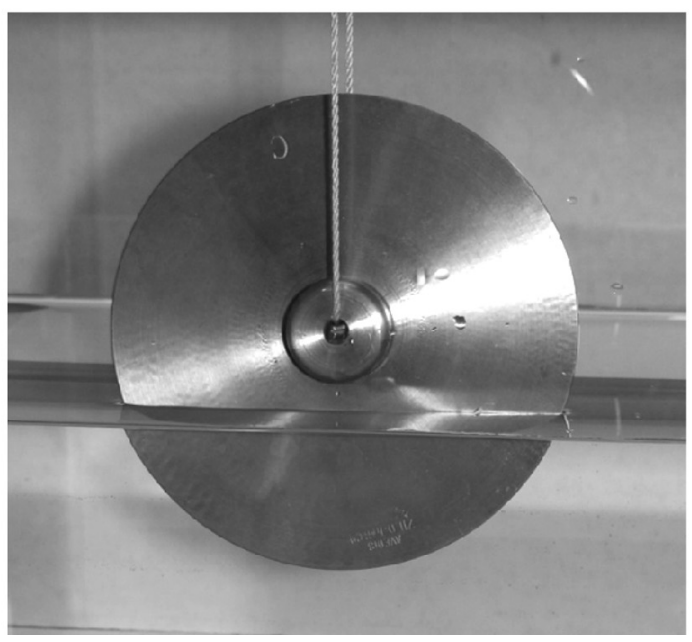

$$
\psi_{m n}(r, \theta)=f_{m n}(r)\left\{\begin{array}{c}
\cos \left[m\left(\theta+\phi_{m n}\right)\right] \\
\sin \left[m\left(\theta+\phi_{m n}\right)\right]
\end{array}\right\} .
$$

The circular symmetry dictates that the eigenfunctions may be designated by the integers $(m, n)$, where $m$ represents the number of nodal diameters and $n$ the number of nodal circles. For a flat disk, the radial functions $f_{m n}(r)$ involve known linear combinations of Bessel functions (see, e.g., Ref. 2, pp. 78-80). With the added boss in the center, an analytic solution for the crotale modes does not exist, but the angular form will still be as shown in Eq. (1). As a result, any crotale mode possessing at least one nodal diameter is two-fold degenerate, corresponding to the cosine and sine solutions of Eq. (1). Each degenerate mode pair consists of complementary spatial patterns, rotated by $\pi / 2 m$ with respect to each other (i.e., with nodes and antinodes interchanged).
When mounted on stands for conventional performance (i.e., with fixed center), the steady state sound of the crotale is dominated by the fundamental mode $(2,0)$, which determines the perceived pitch, along with the $(3,0)$ and $(4,0)$ modes. $^{8}$ The central boss of the crotale serves to tune the overtones such that for the $C_{6}$ crotale the first overtone is an octave above the fundamental and the second overtone is an octave plus a minor seventh, leading to a clear sense of pitch. These relations vary in the higher pitches, as tabulated in Ref. 8, with the second overtone at an octave plus a fifth for several of the higher pitched crotales.

The different fundamental frequencies of the crotales are obtained primarily by varying the diameters of the disks; most sets show little, if any, variation in thickness and boss dimensions over the full range of pitches. 


\section{EXPERIMENAL RESULTS}

Data presented in this section were taken on a $C_{6}$ crotale manufactured by Zildjian [Fig. 1(a)], from a set nominally tuned to an $A_{4}=442 \mathrm{~Hz}$ standard. The $C_{6}$ crotale has a diameter of $13.3 \mathrm{~cm}$ and a thickness of $4.7 \mathrm{~mm}$. The central boss has a diameter of $29.3 \mathrm{~mm}$ and a height of $13.1 \mathrm{~mm}$. The hole through the center has a diameter of $6.4 \mathrm{~mm}$. There is also a rounded central cutout on the side opposite the knob to accommodate a mounting grommet and nut. This cutout has a diameter of $16.8 \mathrm{~mm}$ at the surface and extends to a depth of $5.8 \mathrm{~mm}$, where it joins the center hole. This crotale has a mass of $646 \mathrm{~g}$.

\section{A. Crotale modes in air}

Frequencies and mode shapes of the crotale were obtained using time-averaged electronic speckle-pattern interferometry (ESPI) as described by Moore et al. ${ }^{17-20}$ Initial data were collected for the crotale in air, mounted rigidly with a bolt through the center hole in a manner similar to that used in conventional performance. The crotale was excited acoustically using a speaker driven by a function generator.

Figure 2(a) shows ESPI images of the first eight mode pairs along with their $(m, n)$ designations. The vibrational patterns of the pairs are orthogonal to one another, as expected. As reported by Deutsch et al. ${ }^{8}$ it was determined that only the first three mode pairs sustain well after the crotale is struck, with the fundamental ringing for the longest time by far. The higher modes shown in Fig. 2 contribute to the initial attack transient of the sound. The relative amplitudes of these modes vary with the location of the strike, hardness of the mallet, etc. and are not considered here.

Figure 2(b) shows data for additional acoustically significant modes that are present when the crotale is suspended freely, by string through the center hole. This suspension [see Fig. 1(c)] allows the center of the crotale to vibrate and simulates the performance technique used by most musicians when the water crotales effect is called for. The modes shown in Fig. 2(b) sustain well and can be very prominent in the spectrum depending on the manner in which the crotale is struck. This is especially true of the $(0,1)$ mode, which is clearly heard unless the strike point falls on the nodal circle. If the $C_{6}$ crotale is struck on the central boss, the $(0,1)$ mode is heard more clearly than even the fundamental, and the perceived pitch is that of the $(0,1)$ mode. To our knowledge the central boss is not used as a strike point in practice; nonetheless the $(0,1)$ mode does provides a strong overtone when the crotale is freely suspended.

The absence of the $(1,1)$ mode when the crotale is mounted on a stand is initially puzzling. One might expect that clamping the center would not inhibit any mode with a nodal diameter. However, Fig. 2(b) shows that the $(1,1)$ mode involves more motion near the center than the modes containing multiple diameters shown in Fig. 1(a). In particular, the $(1,1)$ mode requires portions of the central boss to oscillate (note the interference fringes that extend into the region of the boss). When the center is tightly clamped, this motion is inhibited. a)

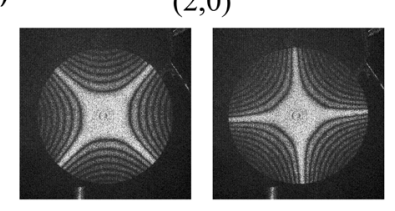

$(4,0)$

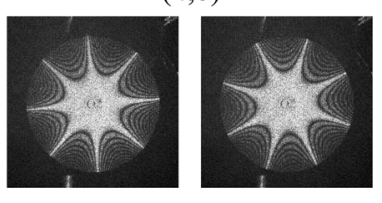

$(2,1)$

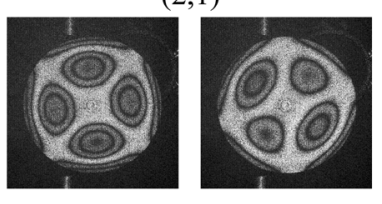

$(3,1)$

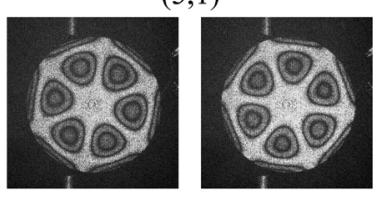

b)

$(0,1)$

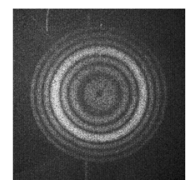

FIG. 2. ESPI images of $C_{6}$ crotale modes in air. Nodal regions appear white; gray lines represent contours of equal amplitude surface motion. (a) First eight mode pairs with standard mount (clamped center). (b) Three additional modes when freely suspended.

Table I lists the frequencies of all the modes shown in Fig. 2, along with the overtone ratios relative to the $(2,0)$ fundamental. For mode pairs, the percentage of frequency splitting is also given. The symmetry of the crotale and the mounting technique may be judged by the symmetry of the nodal patterns as well as by the small amount of frequency splitting observed for each (nearly) degenerate mode pair. In all but one case this splitting amounts to less than $1 \%$ of the mean frequency of the pair.

\section{B. Crotale modes in water}

To record the mode shapes and frequencies in water, the crotale was mounted inside an aquarium tank. Water was added to the tank in small increments so that data could be taken at small depth intervals ranging from zero (i.e., no water) to fully submerged [see Fig. 1(c)]. The driving speaker remained in the air, but had to be carefully positioned to drive the higher order crotale modes at the larger water depths.

Most of the measurements were taken in a glass aquarium tank of approximate dimensions $35 \times 20 \mathrm{~cm}$, with a height of about $24 \mathrm{~cm}$. This tank reasonably approximates 
TABLE I. $C_{6}$ crotale mode frequencies in air obtained using electronic speckle-pattern interferometry. The third column shows the percentage of frequency splitting for each mode pair. The fourth column shows the overtone ratio relative to the fundamental $(2,0)$ mode.

\begin{tabular}{lccc}
\hline \hline Mode $(m, n)$ & Frequency $(\mathrm{Hz})$ & $\Delta f / f_{\text {avg }}(\%)$ & $\left(f / f_{1}\right)_{\text {avg }}$ \\
\hline$(2,0)$ & 1052,1055 & 0.3 & - \\
$(0,1)$ & 1630 & - & 1.55 \\
$(3,0)$ & 2133,2146 & 0.6 & 2.03 \\
$(1,1)$ & 3398,3408 & 0.3 & 3.23 \\
$(4,0)$ & 3688,3722 & 0.9 & 3.52 \\
$(5,0)$ & 5582,5681 & 1.8 & 5.34 \\
$(2,1)$ & 6902,6926 & 0.3 & 6.56 \\
$(6,0)$ & 7869,7896 & 0.3 & 7.48 \\
$(3,1)$ & 9319,9367 & 0.5 & 8.87 \\
$(7,0)$ & 10415,10432 & 0.2 & 9.89 \\
\hline \hline
\end{tabular}

the size of container that may be used in performance (the type and size of water container are not specified in the musical scores when the water crotale effect is called for.) Experimental results were not sensitive to the specific tank dimensions.

\section{Mode shapes in water}

Figure 3(a) shows ESPI images and frequencies at a representative water depth of $5 \mathrm{~cm}$ for the clamped mount modes corresponding to Fig. 2(a) (note the horizontal water line visible just below the center hole). Figure 3(b) shows the additional modes of the freely suspended crotale, also at a $5 \mathrm{~cm}$ depth, corresponding to those of Fig. 2(b). These images show that any mode pairs that were not initially oriented relative to the horizontal are now aligned with respect to the water perturbation. This is particularly clear in the images of the two $(1,1)$ modes shown in Figs. 2(b) and 3(b). In Fig. 3(b) the nodal diameters have become oriented horizontally and vertically in response to the water.

It is also clear that for the most part the mode shapes are not significantly changed by the presence of the water. This is true at all of the recorded depths, although there are occasional images [such as the lower frequency $(2,1)$ mode] which look unusual. These appear to be simply cases where the image was difficult to obtain, rather than part of a pattern.

An interesting effect was observed due to the presence of the water as an optical medium in the interferometer. Due to the index of refraction of the water $(n=1.33=4 / 3)$ the optical path difference in water is larger than that in air by a 4:3 ratio, for a given amount of surface motion. As a result, the images (see Fig. 3) show more contours under water than in air, and there are often discontinuities visible at the water line. These are purely optical effects and do not indicate anything unusual in the actual motion of the crotale.

\section{Frequencies in water}

It is well known in the engineering literature that vibrational frequencies of plates decrease in water, ${ }^{12-16}$ and this is clearly the case with the crotale. Figure 4 is a plot of frequency vs water depth for the ten modes shown in Figs. 2 a) $(2,0)$

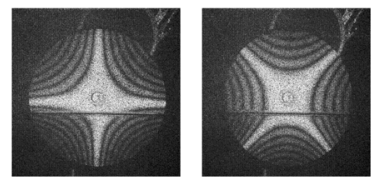

$(4,0)$

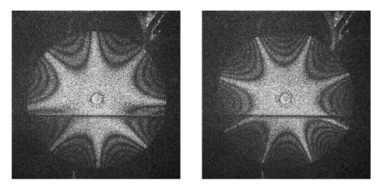

$(2,1)$

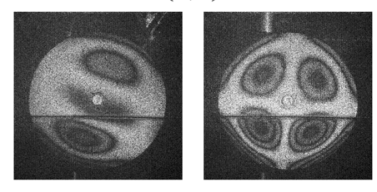

$(3,1)$
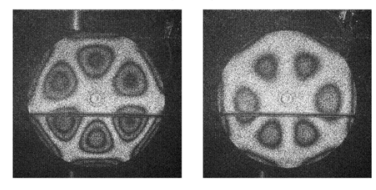

$(0,1)$

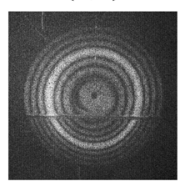

FIG. 3. ESPI images of $C_{6}$ crotale modes while partially submerged in water $(d=5 \mathrm{~cm})$. Horizontal water line is visible just below the middle of the crotale. (a) Clamped at center. (b) Freely suspended.

and 3. All of the modal frequencies decrease monotonically with depth, in agreement with the general perception of a downward glissando when the effect is heard. Frequencies of the mode pairs have been averaged for this graph as their splitting is not clearly visible on this scale. The frequency splitting of individual mode pairs in response to the water perturbation is discussed below.

In addition to the perceived decrease in pitch, the data also show that the overtone ratios (and thus the timbre of the sound) change with depth. This is caused by the fact that different modes decrease in frequency by different percentages as the crotale is submerged. Figure 5 shows the general trend that higher frequency modes are less affected by the water, with the fundamental $(2,0)$ mode lowered by the largest percentage. For modes with $n=0$, the water effect decreases monotonically with increasing number of nodal diameters.

Figure 6 shows the overtone ratios of the four acoustically significant modes (when freely suspended) relative to the fundamental $(2,0)$, as a function of water depth. For the mode pairs, the two frequencies have been averaged in this figure. When fully submerged, these overtone ratios increase in the range of one half to one full semitone over their values in air. The change in perceived timbre is thus rather small in 


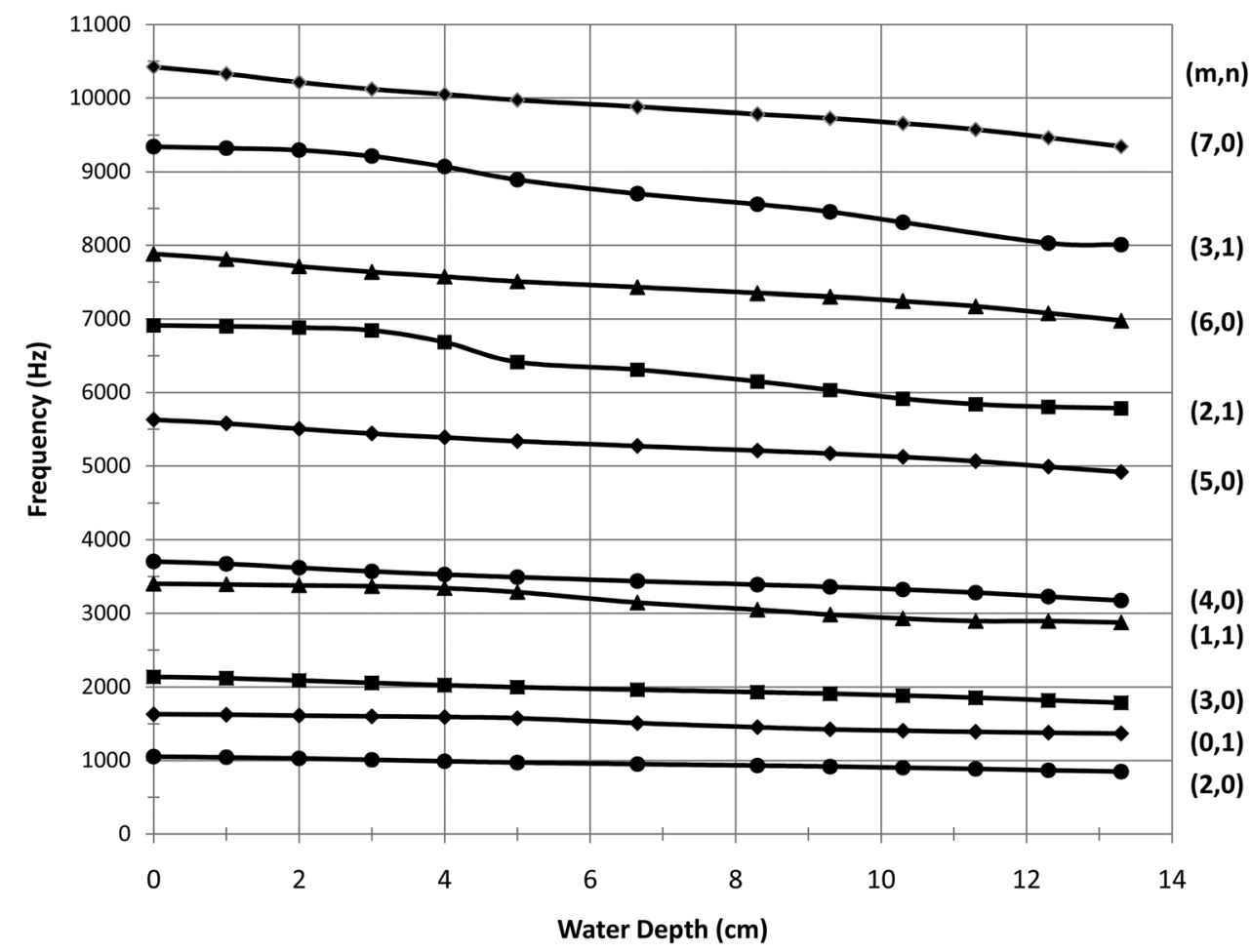

FIG. 4. Frequency vs water depth for $C_{6}$ crotale modes shown in Figs. 2 and 3 . The mode pair frequencies have been averaged, as the splitting is not clearly visible on this scale. Mode designations $(m, n)$ are shown to the right.

practice, particularly as the crotale is not fully submerged during performance.

The splitting of degenerate modes due to the presence of the water perturbation is a particularly interesting effect, which can lead to the production of audible "beats",22 under certain circumstances. Figure 7(a) shows the frequencies of the $(2,0)$ fundamental mode pair vs water depth. The frequencies of the two modes (distinguished visually by horizontal and vertical nodal lines in one case vs diagonal nodal lines in the other) split, come back together, and then reverse roles, with maximum splitting occurring at four depths. A simple model that explains this pattern of splitting and reversal is described in Sec. IV. The maximum pitch decrease of the fundamental mode corresponds to 373 cents, which is a bit less than a major third (400 cents). The maxi- mum frequency difference for this mode pair is $\Delta f=28 \mathrm{~Hz}$. If the crotale is struck such that both modes are excited in roughly equal amounts, beats can be heard at some depths, corresponding to frequency differences of up to about $15 \mathrm{~Hz}$.

Similar frequency splitting occurs for all of the degenerate modes, with the number of frequency difference maxima equal to twice the number of nodal diameters $(2 \mathrm{~m})$ as discussed in Sec. IV. Figure 7(b) shows the frequency splitting of the $(1,1)$ mode pair. For this pair the frequencies split twice, with a maximum frequency difference of $\Delta f=117 \mathrm{~Hz}$. The pitch decrease is slightly more than a minor third (308 cents) when fully submerged.

The data discussed above were taken using a $C_{6}$ crotale, which is the lowest available pitch, and thus the largest diameter disk. Figure 8 shows the maximum percentage

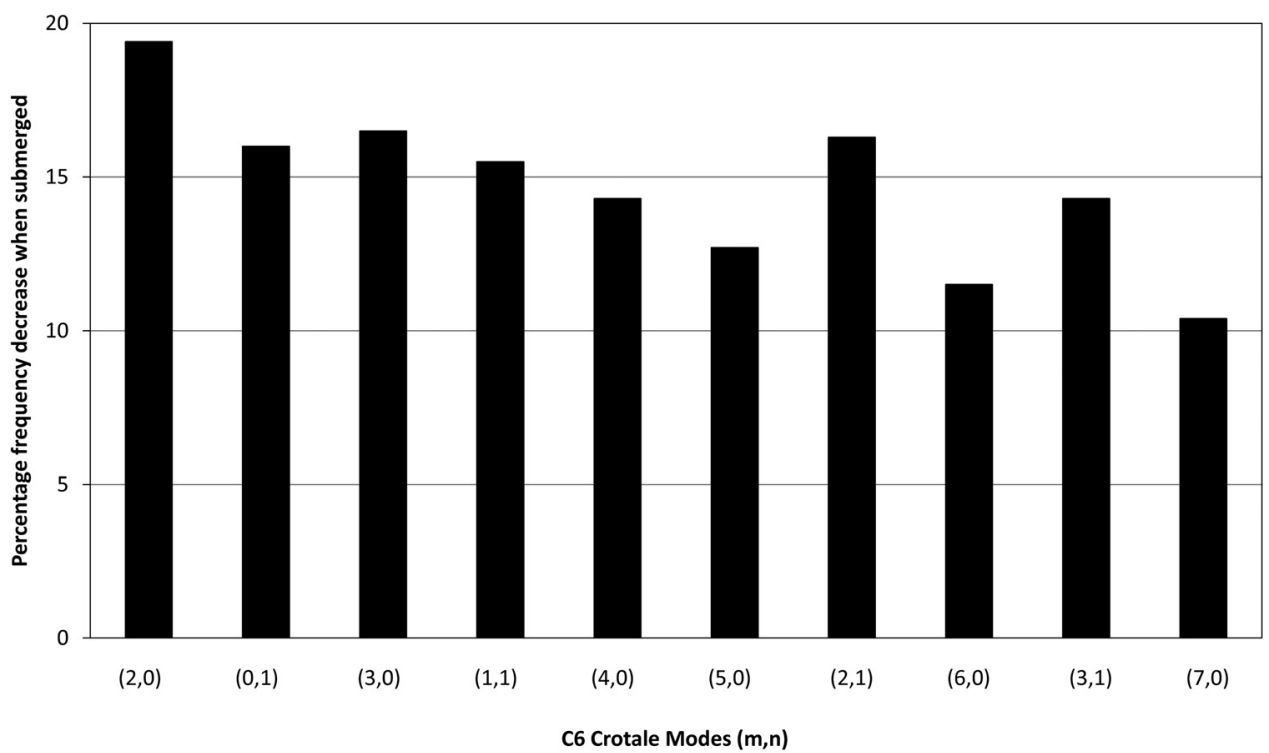

FIG. 5. Percentage of frequency decrease for first ten modes of the $C_{6}$ crotale, when fully submerged in water. Frequencies of mode pairs have been averaged for this plot. Higher order modes are generally less affected by the water. 
frequency drop of the fundamental $(2,0)$ mode for six different crotales spanning the full range from $C_{6}$ to $F_{8}$. Although the $F_{8}$ crotale is taken from a Paiste set (Zildjian does not make crotales above $C_{8}$ ), the graph clearly shows the general trend that the percentage of frequency drop (and thus the perceived pitch drop) decreases for higher pitched crotales. This result is discussed in Sec. IV.

\section{ANALYSIS AND DISCUSSION}

\section{A. Fluid structure interaction}

When a vibrating plate is submerged, the water that must move with it can be treated as adding virtual mass to the plate, without altering its stiffness. From an energy standpoint, the water acts to increase the kinetic energy without changing the potential energy of the system. As a result, the natural frequencies decrease when the plate is submerged. In a quantitative analysis, the basic problem is to calculate the increased inertia due to the added virtual mass of the water moving in response to the plate. When the added virtual mass is known, the decrease in plate frequency can be determined.

The "assumed modes" approach used to calculate the modal frequencies of a disk in water (see, e.g., Kwak, Ref. 13 ) is outlined briefly here. This method is applicable when the mode shapes in water are approximately equal to the mode shapes in air. The speckle pattern images (see Figs. 2 and 3 ) indicate that this is a reasonable approach with the crotale, even though we do not have an analytic solution for these modes. Although quantitative calculation requires knowledge of the normal mode eigenfunctions, several qualitative comparisons with the data can be made based on the general results of this assumed modes approach.

Treating the water as an incompressible fluid, Rayleigh's quotient can be used to relate the frequencies in air and water to the potential and kinetic energies of the system as follows. The frequency in air is

$$
f_{a}^{2} \propto\left(\frac{V_{p}}{T_{p}^{*}}\right)_{\text {air }}
$$

where $f_{\mathrm{a}}$ is the natural frequency of a plate mode in air. $V_{p}$ represents the maximum potential energy and $T_{p}^{*}=T_{\max } / \omega^{2}$ is the reference kinetic energy of the plate. In water, the relation becomes

$$
f_{w}^{2} \propto\left(\frac{V_{p}}{T_{p}^{*}+T_{w}^{*}}\right)_{w a t e r},
$$

where $f_{w}$ is the natural frequency in water and $T_{w}^{*}$ represents the reference kinetic energy of the water due to the motion of the plate. As the maximum potential energy is unchanged by the presence of water, Eqs. (2) and (3) can be combined to yield

$$
f_{w}=\frac{f_{a}}{\sqrt{\left(1+T_{w}^{*} / T_{p}^{*}\right)}}=\frac{f_{a}}{\sqrt{1+\beta}} .
$$

Thus it is $\beta \equiv T_{w}^{*} / T_{p}^{*}$ the ratio of water reference kinetic energy to plate reference kinetic energy, that determines the lowering of the frequency. For known mode shapes and boundary conditions, $T_{p}^{*}$ and $T_{w}^{*}$ can be determined, although the details of the water calculation are beyond the scope of this paper.

The ratio $\beta$ is called the added virtual mass incremental (AVMI) factor and can be written for each mode $(m, n)$ as

$$
\beta_{m n}=\frac{T_{w}^{*}}{T_{p}^{*}}=\Gamma_{m n}\left(\frac{\rho_{w}}{\rho_{p}}\right)\left(\frac{a}{h}\right),
$$

where $\rho_{w}$ and $\rho_{p}$ are the mass densities of the water and plate respectively, $a$ is the plate radius, and $h$ its thickness. $\Gamma_{m n}$ is called the nondimensionized added virtual mass incremental (NAVMI) factor and is a function of the vibrational mode

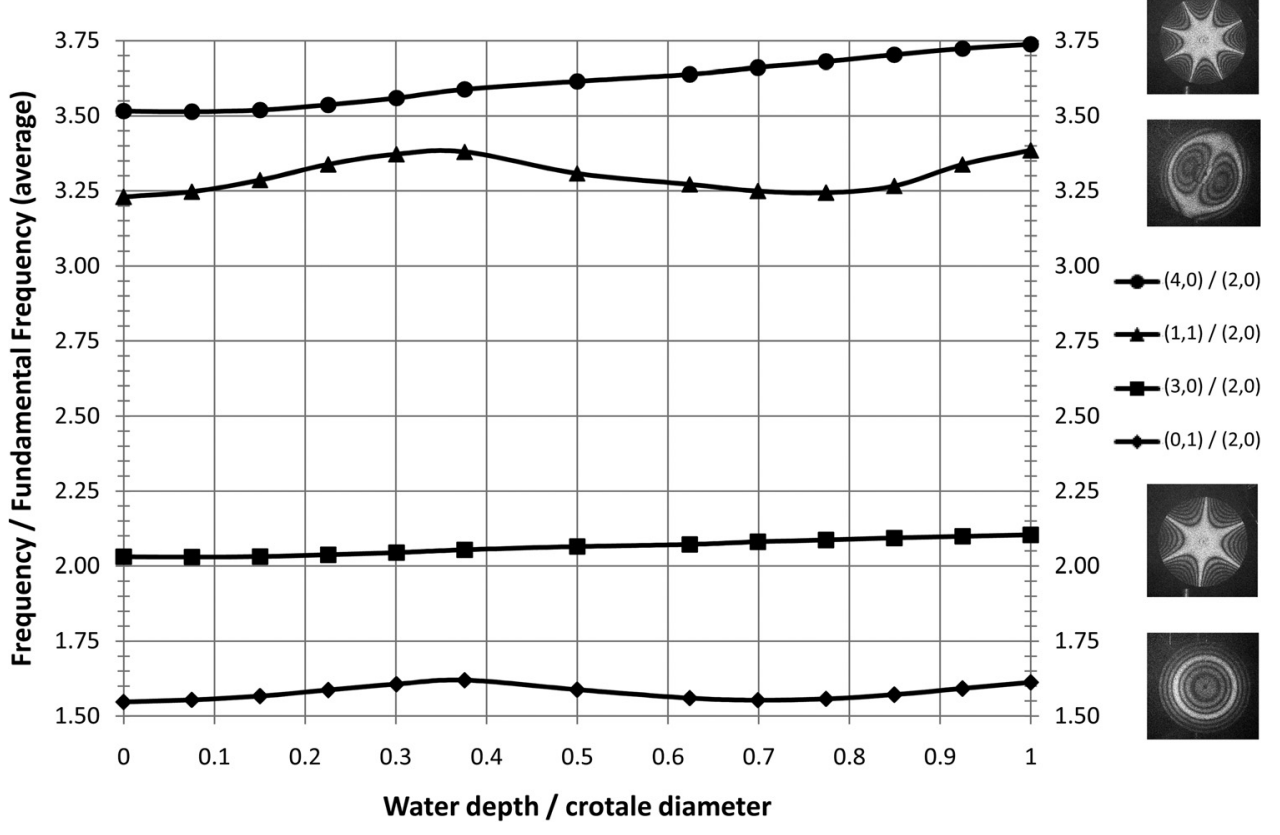

FIG. 6. Overtone ratios vs water depth for $C_{6}$ crotale modes with significant sustain when freely suspended. Mode pair frequencies have been averaged. The overtone ratios tend to increase with depth. 
$(m, n)$ and the boundary conditions. In practice, the quantitative solution comes down to computation of $\Gamma_{m n}$.

Equations (4) and (5) show that all modal frequencies should decrease in water, as reported here (see Fig. 4), and throughout the literature. It is also clear that for a given mode, $\beta$ increases with the ratio $a / h$, in accord with the intuitive notion that a relatively thicker plate should be less affected by the presence of water, while a plate of larger surface area should show a larger effect. This explains the results of Fig. 8, showing that smaller diameter plates (with approximately the same thickness) show a smaller percentage frequency drop (see Ref. 8 for a listing of $C_{6}-C_{8}$ crotale dimensions from a representative Zildjian set).

The results shown in Fig. 5 indicate that lower order crotale modes are influenced more by the presence of water than higher order modes. These results are in qualitative agreement with $\mathrm{Kwak}^{13}$ who calculated that the effect of the added virtual mass of a plate due to water contact generally decreases as the mode order increases. In particular, he showed that for a specified $m$ (or $n$ ) value, the frequency change decreases with increasing $n$ (or $m$ ). These trends were verified experimentally by Amabili et al. ${ }^{21}$ and via calculation and finite element analysis by Jhung et al. ${ }^{14}$ Lamb (see Ref. 10, p. 209) provides the following physical interpretation: "In the case of ... a plate vibrating in segments separated by nodal lines, the effect of the inertia of the water will be less, owing to the freedom of lateral motion near the surface, between adjacent segments in opposite phases."

\section{B. Qualitative model of mode splitting effects}

The behavior of the mode pairs shown in Figs. 7(a) and 7(b) requires a model that accounts for the added virtual

a)

$(2,0)$ modes

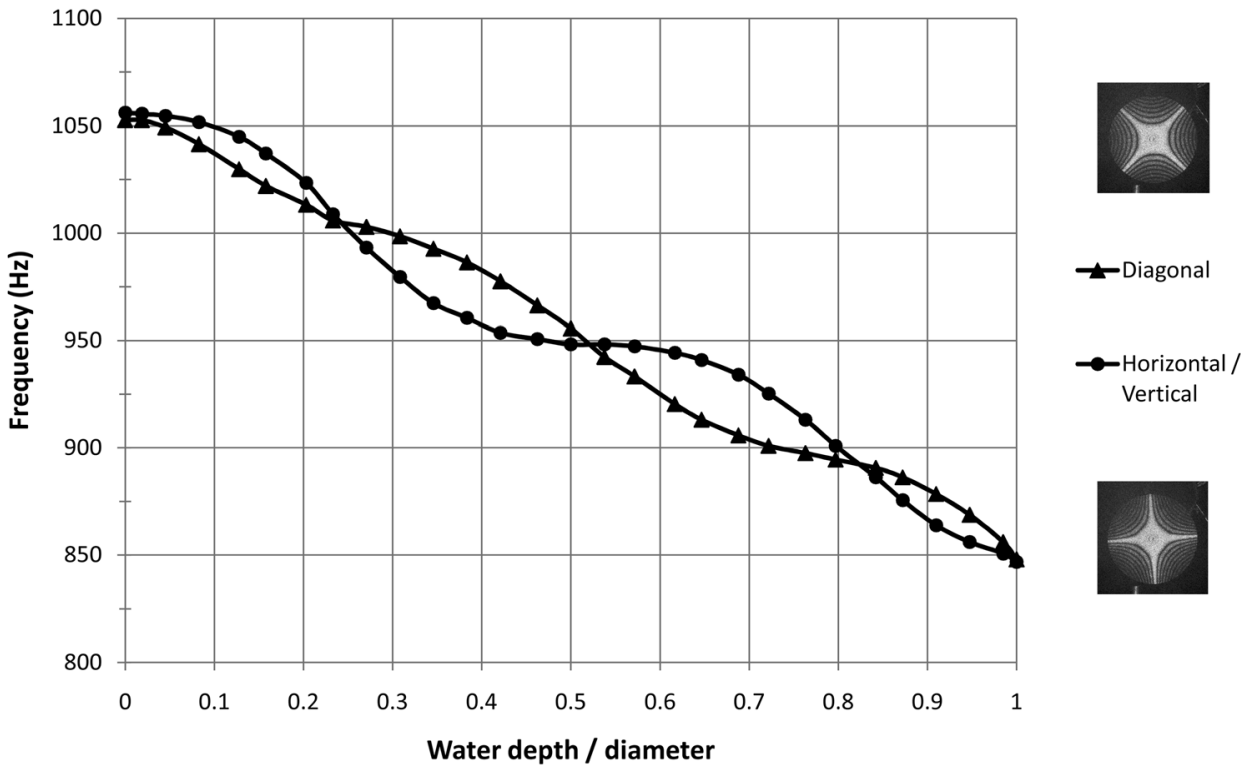

b)

$(1,1)$ modes

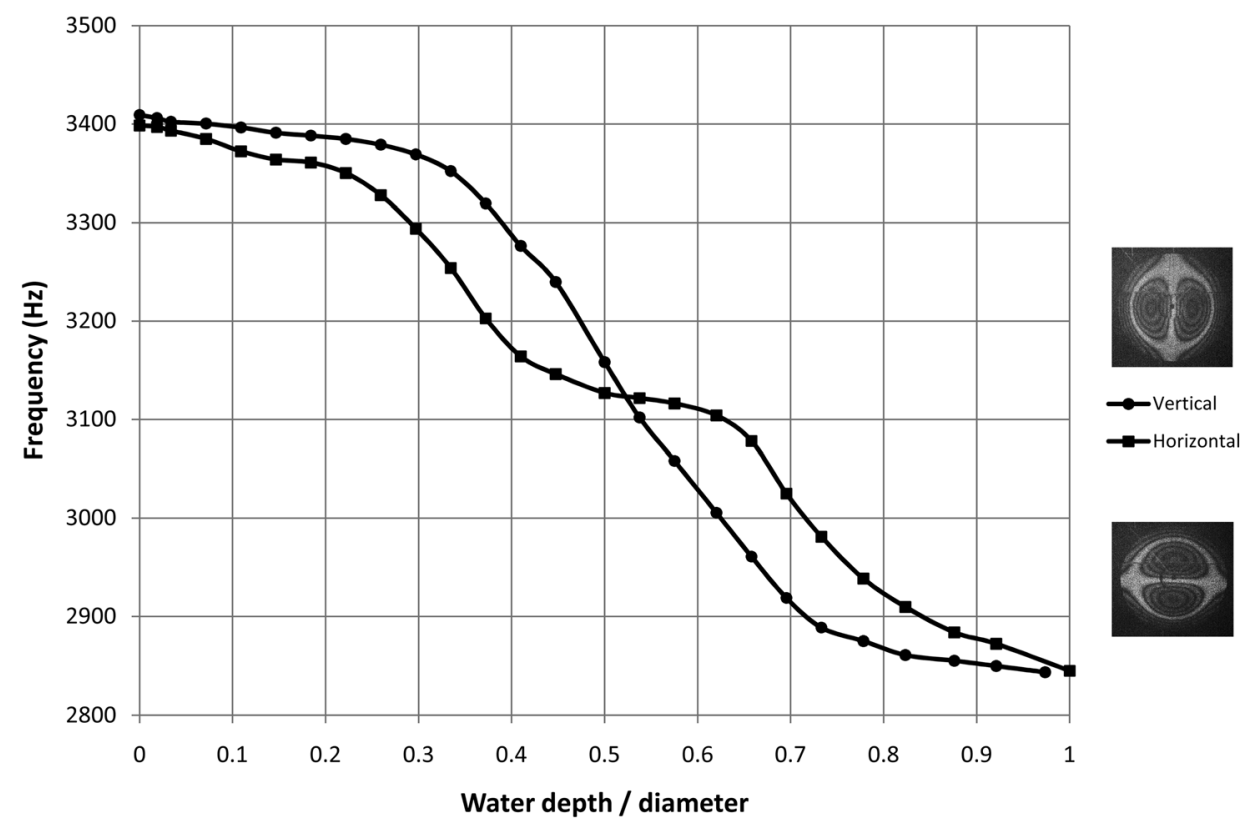

FIG. 7. Frequency vs depth for modes with $m=2$ and $m=1$. For mode pairs, the frequency splits $2 \mathrm{~m}$ times. (a) Fundamental $(2,0)$ mode. Note that the degeneracy of the two modes is lifted and then reestablished four $(2 \mathrm{~m})$ times as the water depth is increased. Maximum frequency splitting is $\Delta f=28 \mathrm{~Hz}$. Maximum pitch decrease is 373 cents (400 cents corresponds to a major third). (b) The (1,1) mode. The degeneracy is lifted and reestablished two times. Maximum splitting is $\Delta f=117 \mathrm{~Hz}$. Maximum pitch decrease is 308 cents (just over a minor third). 


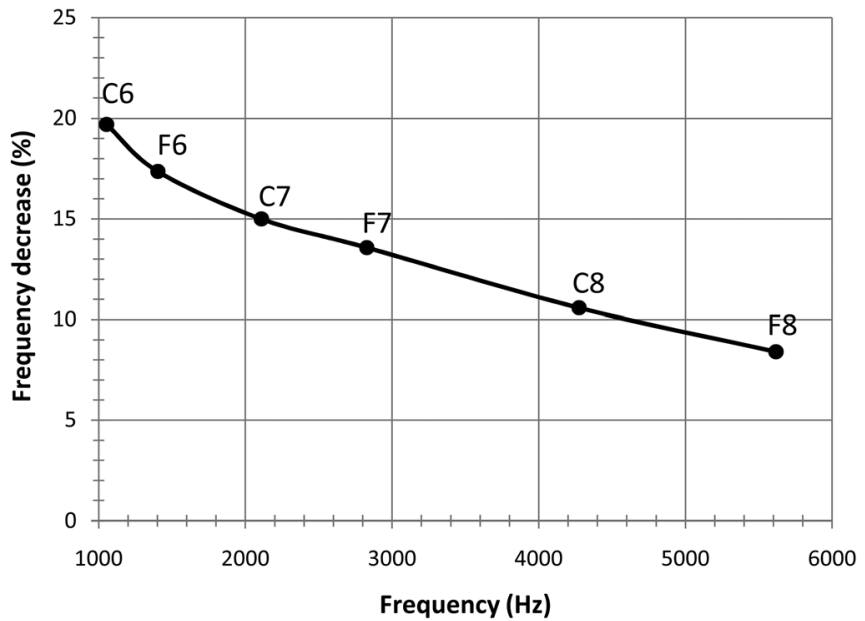

FIG. 8. Maximum percentage frequency decrease (fully submerged) of fundamental mode vs crotale pitch, for five Zildjian and one Paiste $\left(F_{8}\right)$ crotales. The water effect is largest for the lowest pitched crotale.

mass as a function of water depth. In the present analysis we examine a simple qualitative model that can provide a physical understanding of the observed data.

\section{Conceptual model}

Assuming that water adds virtual mass to the crotale, without altering its stiffness, the water can only act to lower the natural frequencies. For simplicity we consider only a small layer of water in contact with the crotale surface. Added water mass at a node should have no effect on the frequency; for other regions of the mode shape the frequency lowering effect should increase with proximity to an antinode, where the additional kinetic energy of the added mass is greatest. Thus, the two modes of a pair may be affected differently at a particular depth, due to the orientation of their nodal and antinodal regions below the water line. According to Rayleigh's principle, the two modes of an initially degenerate pair will orient themselves relative to the water perturbation such that one has the lowest frequency and the other the highest frequency possible.

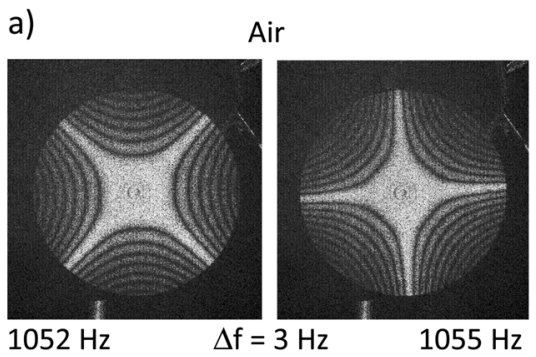

b)

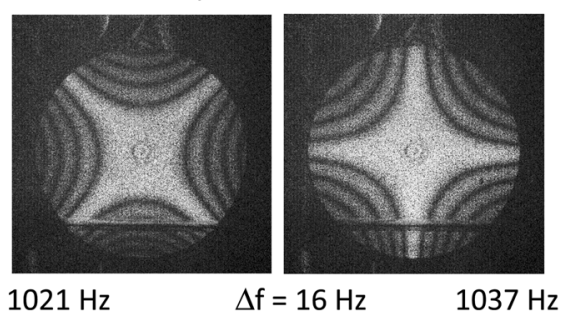

c)

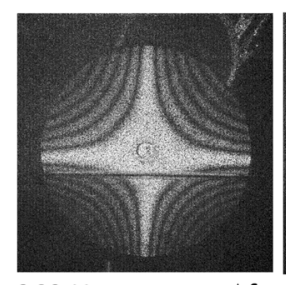

$962 \mathrm{~Hz}$
The ESPI images of Fig. 9 illustrate this effect for the fundamental $(2,0)$ mode of the $C_{6}$ crotale. In Fig. 9(a), the nearly degenerate pair is shown in air, with frequencies differing by only $3 \mathrm{~Hz}$ (about $0.3 \%$ ). At a water depth of $d=2 \mathrm{~cm}$ [Fig. 9(b)] both frequencies have decreased, and the splitting has increased to $\Delta f=16 \mathrm{~Hz}$. The mode on the left, with diagonal nodal regions, shows a region of greater amplitude in contact with the water, and thus a lower frequency, than the mode on the right. Figure 9(c) shows that at a depth of $d=5 \mathrm{~cm}$ the situation has reversed. Both frequencies have continued to decrease, and the splitting is now $\Delta f=22 \mathrm{~Hz}$, but the mode with diagonal nodal regions (on the right) now produces the higher frequency. At this depth the diagonal orientation appears to have less integrated amplitude over the area of water contact and thus its frequency is higher than the companion mode. An equivalent approach is to visually judge the amount of nodal area (which will be unaffected by the water) beneath the water line in each orientation. The vibrational pattern with the larger submerged nodal area will display the higher of the two frequencies. This may be confirmed in both Figs. 9(b) and 9(c).

It is clear from symmetry that the two modes of the $(2,0)$ pair should ideally be degenerate in air and at depths corresponding to half, and fully submerged. It is less obvious that there are two other depths [see Fig. 7(a)] for which these two orientations of the $(2,0)$ mode are degenerate. The geometry of the integrated water motion (or, alternatively, the nodal area estimation) yields the result that the number of maximum frequency splittings is equal to twice the number of nodal diameters $(2 \mathrm{~m})$. Qualitatively, as the number of nodal diameters increases there are more depths at which the two orientations produce the same integrated amount of added virtual mass.

\section{Numerical test of the model}

To test this geometrical hypothesis, a numerical calculation was performed. As seen in Eq. (4), the frequency change of a given mode depends on the ratio of the reference kinetic energies of the water and the plate, $T_{w}^{*} / T_{p}^{*}$. For the plate, the reference kinetic energy is given by (see Ref. 13) 

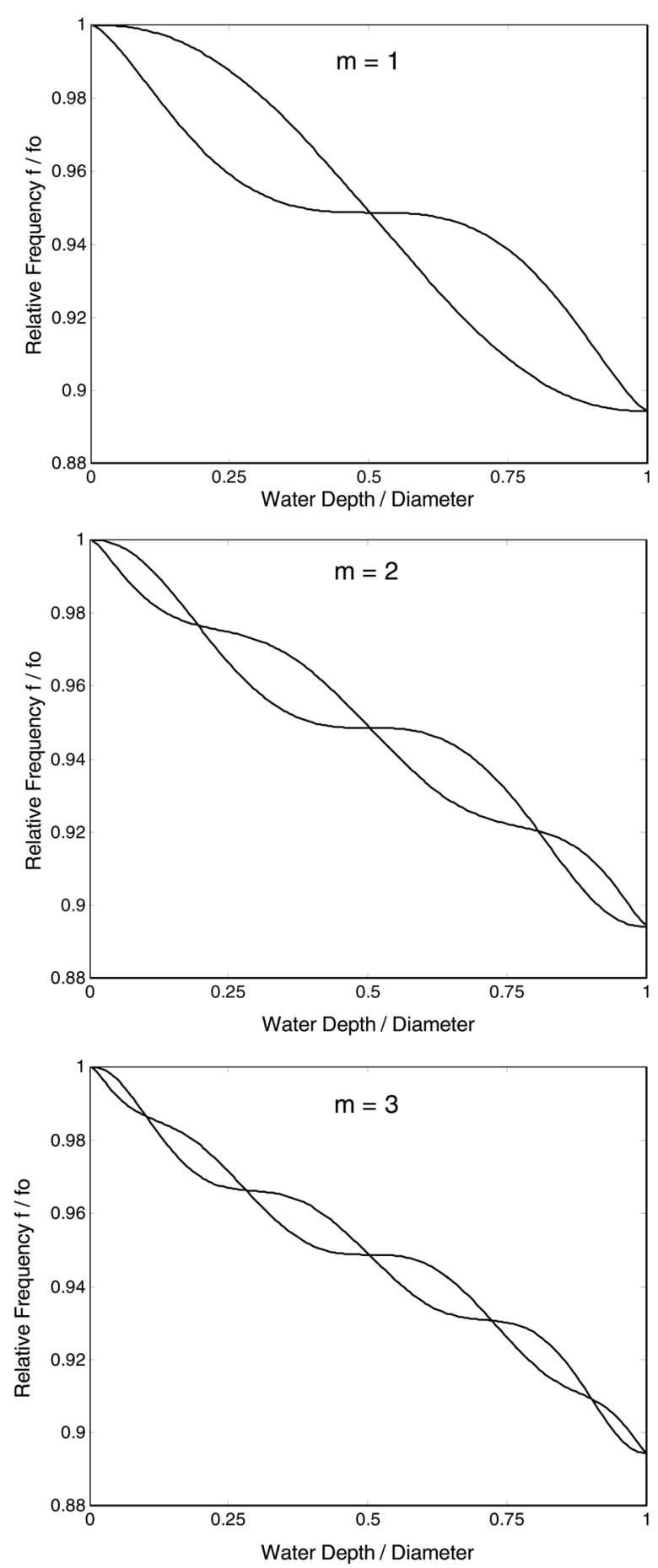

FIG. 10. Numerical plots showing the relative frequency vs water depth for three mode pairs ( $n=0$ in each case) based on the qualitative model of Sec. IV. Note that the number of frequency splittings is equal to $2 m$ in each case. Compare with the experimental data shown in Fig. 7.

$$
T_{p}^{*}=\frac{1}{2} \rho_{p} h \int_{\mathcal{A}_{0}} \psi^{2} d A .
$$

Thus, the reference kinetic energy is proportional to the square of the wave function integrated over the entire surface area $\mathcal{A}_{0}$ of the plate. To obtain a qualitative expression for the reference kinetic energy of the water, we treat a thin uniform layer of water in contact with the plate, resulting in

$$
T_{w}^{*} \propto \int_{\mathcal{A}} \psi^{2} d A
$$

where the integration is only over the area $\mathcal{A}$ of the plate covered by water (i.e., the portion of the plate below the water line at a given depth, $d$ ). In this way the qualitative behavior of the added virtual mass incremental (AVMI) factor $\beta_{m n}=T_{w}^{*} / T_{p}^{*}$ as a function of water depth may be determined:

$$
\beta_{m n}(d)=\frac{T_{w}^{*}}{T_{p}^{*}}=\frac{\int_{\mathcal{A}} \psi_{m n}^{2} d A}{\int_{\mathcal{A}_{0}} \psi_{m n}^{2} d A} .
$$

For the eigenfunction $\psi_{m n}$, we use both the cosine and sine angular functions of Eq. (1), with $\phi_{m n}$ set to zero for alignment of the modes relative to the water line. For the radial functions $f_{m n}(r)$, polynomials are used as trial functions. As in McLachlan's paper, ${ }^{11}$ we use $f_{m n}(r)=(r / a)^{m}$, and treat only the $n=0$ (no nodal circles) case. Figure 10 shows the resulting plots of the relative frequency $f / f_{o}$ vs water depth for three representative values of $m$. These results are consistent with the simple model described above and with the experimental data shown in Fig. 7. The qualitative structure of these curves is not sensitive to the choice of radial function, but depends geometrically on the number of diameters $(m)$ in the mode.

A detailed calculation, as well as finite element analysis, of the frequency change vs water depth is presented by Jeong et al. ${ }^{15}$ for the case of a disk with a clamped edge, in contact with water on one side. Our model of mode splitting in water is in qualitative agreement with the results of Jeong et al. (see Figs. 6 and 7 of Ref. 15), although the detailed shapes of his frequency vs water depth plots differ from ours due to differences in plate geometry and boundary conditions.

From a musical standpoint, the practical result of the degenerate mode splitting is that in addition to pitch and timbre changes as the crotale is submerged, there is the potential for audible beats to occur at particular depths.

\section{CONCLUSIONS}

The mode shapes and frequencies of a $C_{6}$ crotale have been measured as a function of water depth. The mode shapes are seen to remain essentially unchanged, while all the natural frequencies decrease monotonically with depth. The data presented in Sec. III show that the lower frequency modes are generally affected by the water the most, with the fundamental frequency lowered by the largest percentage. Thus, the perceived pitch drops and the timbre changes slightly when the musical water crotale effect is produced.

All crotale modes containing at least one nodal diameter are ideally doubly degenerate. This degeneracy can be lifted by the water perturbation, leading to frequency splitting that varies with depth. This splitting of degenerate modes, especially the fundamental $(2,0)$ mode, can lead to audible beats at some water depths. A qualitative model is presented in Sec. IV B to explain the behavior of the degenerate modes in response to the water perturbation. 
In addition to the $C_{6}$ crotale, the maximum frequency decrease in water was measured for crotales of several different pitches. Figure 8 shows that the pitch decrease is smaller for the higher pitched (smaller diameter) crotales. The maximum pitch decrease for the lowest crotale $\left(C_{6}\right)$ is nearly a major third (373 cents), while the maximum pitch drop of the highest crotale $\left(F_{8}\right)$ is between one and two semitones (152 cents). These results are in qualitative agreement with models that treat the water as a perturbation acting to add virtual mass to the crotale without altering its stiffness.

The method of suspension that is used when producing the water crotale effect has also been examined and shown to result in acoustically significant $(0,1)$ and $(1,1)$ modes that are not typically heard when the crotales are mounted on a stand. These modes join the fundamental $(0,2)$ and the $(3,0)$ and $(4,0)$ modes in contributing to the steady state sound of the freely suspended crotale.

\section{ACKNOWLEDGMENTS}

The author would like to thank percussionist Amy Putnam of the University of Puget Sound School of Music for information about the water crotale technique, and for the loan of several crotales. The author also thanks Puget Sound physics colleague Alan Thorndike for many helpful discussions.

${ }^{1}$ Two-octave crotale sets are manufactured by cymbal companies including Zildjian, Sabian, and Paiste. Paiste also sells a 2 1/2 octave set.

${ }^{2}$ E.g., John Cage compositions First Construction (in Metal) (1939), Second Construction (1940), Double Music, with Lou Harrison (1941), and Imaginary Landscape No. 2 (1940).

${ }^{3}$ Star Trek, The Motion Picture, musical score by Jerry Goldsmith (1979).

${ }^{4}$ Stained Glass, composed by David Gillingham (1991).
${ }^{5}$ Symphony No. 2, composed by David Maslanka (1985). An excellent example of the water crotales effect occurs near the end of the second movement, in a recording by the University of Arizona Wind Ensemble, conducted by Gregg Hanson, on Albany Records (1998).

${ }^{6} \mathrm{~N}$. Fletcher and T. Rossing, The Physics of Musical Instruments, 2nd ed. (Springer-Verlag, New York, 1998), pp. 78-80.

${ }^{7}$ T. Rossing, Science of Percussion Instruments, (World Scientific, Hackensack, NJ, 2000), pp. 102-103.

${ }^{8}$ B. M. Deutsch, C. L. Ramirez, and T. R. Moore, "The dynamics and tuning of orchestral crotales," J. Acoust. Soc. Am. 116, 2427-2433 (2004).

${ }^{9}$ Lord Rayleigh, Theory of Sound, 2nd ed. (Macmillan, London, 1894) (reprinted by Dover, New York, 1945), Vol. II, Sec. 302, pp. 162-169.

${ }^{10}$ H. Lamb, "On the vibrations of an elastic plate in contact with water," Proc. R. Soc. London, Ser. A 98, 205-216 (1920).

${ }^{11}$ N. W. McLachlan, "The accession to inertia of flexible discs vibrating in fluid,” Proc. Phys. Soc. 44, 546-555 (1932).

${ }^{12}$ M. K. Kwak and K. C. Kim, "Axisymmetric vibration of circular plates in contact with fluid," J. Sound Vib. 146, 381-389 (1991).

${ }^{13}$ M. K. Kwak, "Vibration of circular plates in contact with water," J. Appl. Mech. 58, 480-483 (1991).

${ }^{14}$ M. J. Jhung, Y. H. Choi, and H. J. Kim, "Natural vibration characteristics of a clamped circular plate in contact with fluid," Struct. Eng. Mech. 21 169-184 (2005).

${ }^{15}$ K. H. Jeong, G. M. Lee, and T. W. Kim, "Free vibration analysis of a circular plate partially in contact with a liquid," J. Sound Vib. 324, 194-208 (2009).

${ }^{16}$ Y. Kozlovsky, "Vibration of plates in contact with viscous fluid: Extension of Lamb's model,” J. Sound Vib. 326, 332-339 (2009).

${ }^{17}$ T. R. Moore, "A simple design for an electronic speckle pattern interferometer,” Am. J. Phys. 72, 1380-1384 (2004).

${ }^{18}$ T. R. Moore, "Erratum: A simple design for an electronic speckle pattern interferometer," Am. J. Phys. 73, 189 (2004).

${ }^{19}$ T. R. Moore and S. A. Zietlow, "Interferometric studies of a piano soundboard," J. Acoust. Soc. Am 119, 1783-1793 (2006).

${ }^{20}$ T. R. Moore and J. J. Skubal, “Time-averaged electronic speckle pattern interferometry in the presence of ambient motion. Part I. Theory and Experiments," Appl. Opt. 47, 4640-4648 (2008).

${ }^{21}$ M. Amabili, G. Dalpiaz, and C. Santolini, "Free-edge circular plates vibrating in water," Int. J. Anal. Exp. Modal Anal., 10(3), 187-202 (1995).

${ }^{22}$ See e.g., T. D. Rossing, F. R. Moore, and P. A. Wheeler, The Science of Sound (Addison Wesley, San Francisco, 2002), pp. 154-156. 